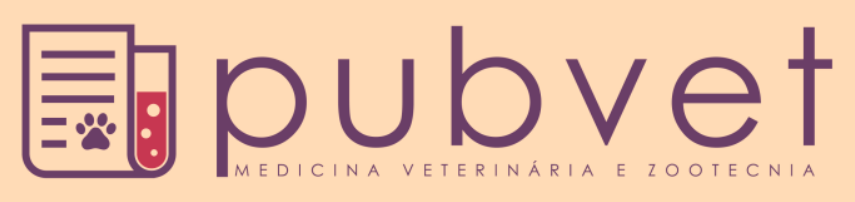

ISSN $1982-1263$

https://doi.org/10.22256/pubvet.v12n6a110.1-5

\title{
Caracterização físico-química de diferentes cortes cárneos de equinos machos
}

\author{
Catarina Nogueira Ribeiro ${ }^{1}$, Cássia Duarte Oliveira $^{2 *} \bullet$, Mauro Ramalho Silva ${ }^{3}$, \\ Harriman Aley Morais ${ }^{4}$
}

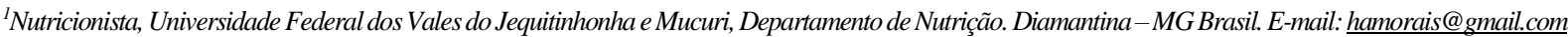
${ }^{2}$ Mestranda do Programa de Pós-Graduação e Ciência e Tecnologia de Alimentos da Universidade Federal dos Vales do Jequitinhonha e Mucuri, Instituto de Ciência e Tecnologia (Diamantina, MG - Brasil).*Autor para correspondência.E-mail:cassiaduartemg2@hotmail.com

${ }^{3}$ Doutorando da Universidade Federal de Minas Gerais, Departamento de Farmácia, Belo Horizonte - MG Brasil. E-mail: mauroramalhosilva@yahoo.com.br ${ }^{4}$ Professor da Universidade Federal dos Vales do Jequitinhonha e Mucuri, Departamento de Ciências Básicas. Diamantina -MG Brasil. E-mail: harriman.morais@ufvjm.edu.br

RESUMO. Objetivou-se determinar a composição centesimal de diferentes cortes cárneos de equinos machos. As amostras dos músculos deltoides (paleta), Longissimus dorsi (contrafilé) e Biceps femoris (coxão duro) foram coletadas em um abatedouro no Estado de Minas Gerais, Brasil. Realizaram-se as análises de umidade, lipídios, proteína e cinzas. As médias encontradas variaram de 77,90 a $78,54 \%$ para umidade, de 1,07 a $1,19 \%$ para lipídios, de 18,39 a 20,64\% para proteína e de 1,12 a 1,77\% para cinzas. Não foram observadas diferenças significativas entre os diferentes cortes cárneos. Concluiu-se, portanto, que a carne dos equinos apresentou baixo teor de lipídios e alto de umidade e proteína, sendo considerada uma carne magra.

Palavras chave: carne de cavalo, contrafilé, coxão duro, físico-química, paleta

\section{Physical-chemical characterization of different meaty cuts of male equines}

ABSTRACT. The objective of this study was to determine the centesimal composition of different meat cuts of male horses. Samples of the deltoid, Longissimus dorsi and Biceps femoris were collected at a slaughterhouse in the State of Minas Gerais, Brazil. Analyzes of moisture, lipids, protein and ashes were carried out. The averages found ranged from 77.90 to $78.54 \%$ for moisture, from 1.07 to $1.19 \%$ for lipids, from 18.39 to $20.64 \%$ for protein and from 1.12 to $1.77 \%$ for There were no significant differences between the different flesh sections. It was concluded, therefore, that the equine meat presented low lipid content and high humidity and protein, being considered a lean meat.

Keywords: horse meat, buckwheat, hard cushion, physicochemical, palette

\section{Caracterización físico-química de diferentes cortes cárnicos de equinos machos}

RESUMEN. Se objetivó determinar la composición centesimal de diferentes cortes cárnicos de equinos machos. Las muestras de los músculos deltoides (paleta), Longissimus dorsi (lomo) y Biceps femoris fueron recolectadas en un matadero en el Estado de Minas Gerais, Brasil. Se realizaron los análisis de humedad, lípidos, proteína y cenizas. Las medias encontradas variaron de 77,90 a $78,54 \%$ para humedad, de 1,07 a $1,19 \%$ para lípidos, de 18,39 a $20,64 \%$ para proteína y de 1,12 a $1,77 \%$ para las cenizas. No se observaron diferencias significativas entre los diferentes cortes cárnicos. Se concluyó, por 
tanto, que la carne de los equinos presentó bajo contenido de lípidos y alto contenido de humedad y proteína, siendo considerada una carne magra.

Palabras clave: carne de caballo, lomo, carnaza cuadrada, físico-química, paleta

\section{Introdução}

Há alguns anos, quando a carne de cavalo começou a ser consumida, o abate de equinos era realizado no final da vida de trabalho desses animais. Isso influenciava diretamente nos atributos de qualidade mais apreciadas pelos consumidores que são o sabor e o valor nutricional (Juárez et al., 2009).

Observando a demanda crescente pelo consumo de carne de cavalo por países europeus, busca-se abater animais mais jovens, que estejam numa faixa entre 11 e 24 meses de idade (Tateo et al., 2008). No ano de 2013, foram produzidas um total de 761 mil toneladas de carne de cavalo e apenas uma pequena porcentagem, 8-10\%, foi abatida, sendo aproximadamente 4,8 milhões (Stanciu, 2015).

De acordo com Costa et al. (2016), o Brasil é um grande criador de equinos, chegando a possuir cerca de 5,5 milhões de cavalos, e por isso é considerado como o país que possui o maior conjunto de equinos da América do Sul e o quinto maior do mundo. Sendo a ordem iniciada pelos EUA e Canadá, seguido pela China, México e Brasil.

Como forma de aumentar o consumo e a produção de carne de cavalo, realizar análises que avaliam sua qualidade é importante. Isso porque existem diferentes músculos que compõe as carcaças dos animais. A utilização na culinária é diferente e influenciada pelo tipo de músculo, podendo ele ser de apoio ou locomotivo, sendo preciso estudar toda a carcaça (Franco \& Lorenzo, 2014).

Assim, considerando que a carne de equinos é um produto de exportação nacional, objetivou-se realizar e avaliar a caracterização físico-química de diferentes cortes cárneos (paleta, contrafilé e coxão duro) de equinos machos.

\section{Material e Métodos}

É necessária uma descrição clara ou uma referência específica original para todos os procedimentos biológico, analítico e estatístico. Todas as modificações de procedimentos devem ser explicadas. Dieta, dados de atividades experimentais se apropriado, animais (raça, sexo, idade, peso corporal, e condição corporal [exemplo, com ou sem restrição de alimentação a água]), técnicas cirúrgicas, medidas e modelos estatísticos devem ser descritos clara e completamente. Informação do fabricante deve ser fornecida na primeira menção da cada produto do proprietário utilizado na pesquisa (para detalhes, ver Produto Comercial). Devem ser usados os métodos estatísticos apropriados, embora a biologia deva ser usada. Os métodos estatísticos comumente utilizados na ciência animal não precisam ser descritos em detalhes, mas as adequadas referências devem ser fornecidas. $\mathrm{O}$ modelo estatístico, classe, blocos e a unidade experimental devem ser designados.

Para a realização deste estudo, contou-se com a colaboração de um abatedouro frigorífico sob SIF, situado um município do Baixo Vale do Jequitinhonha, Minas Gerais, que disponibilizou amostras dos músculos deltóide (paleta), Longissimus dorsi (contrafilé) e Biceps femoris (coxão duro) de animais machos, já abatidos.

As amostras congeladas foram levadas para o Laboratório de Bromatologia da Universidade Federal dos Vales do Jequitinhonha e Mucuri (UFVJM), Diamantina, MG, onde o experimento foi conduzido. Realizou-se a remoção da gordura externa e do tecido conjuntivo visível, e em seguida, os músculos foram cortados e triturados em moedor de carne, sendo armazenadas em potes de PVC, em porções individuais, em um freezer doméstico. Para a realização das análises, essas amostras foram descongeladas em geladeira a 4 ${ }^{\circ} \mathrm{C}$, durante 24 horas.

As análises de umidade (dessecação em estufa a $105 \mathrm{C}$ ), de cinzas (incineração em forno mufla a $550^{\circ} \mathrm{C}$ ), de lipídios (extração em aparelho de Sohxlet) e de proteínas (micro Kjeldhal) foram realizadas de acordo com Brasil (1999). Utilizouse o delineamento experimental inteiramente casualizado (DIC), em blocos, com análise de variância, seguida pelo teste de Tukey a $5 \%$ de significância. Os dados, que correspondem à média de quintuplicatas, foram analisados pelo $\begin{array}{llllll}\text { programa estatístico } & \mathrm{R} & \text { versão } & 2.3 .1 \quad \text { ( }\end{array}$ Development Core Team, 2017).

\section{Resultados e Discussão}

As médias de umidade, lipídeos, proteína e cinzas dos diferentes cortes cárneos (paleta, 
contra-filé e coxão duro), com os respectivos desvios padrão (DP), estão apresentadas na Tabela 1 .

O conteúdo médio de umidade de todos os músculos $(78.28 \%)$ foi similar ao encontrado por Lorenzo et al. (2013a), equivalentes a $77.00 \%$ e por Franco \& Lorenzo (2014), correspondentes a 75.14\%. Em ambos os estudos, os tipos de músculos analisados foram: Longissimus dorsi, Semimembranosus, Semitendinosus, Bíceps femoris, Triceps brachii e Psoas major e minor. Semelhante ao último estudo, não foi observado diferença significativa $(\mathrm{P}>0.05)$ entre os valores de umidade dos diferentes tipos de músculos. Em relação a outras espécies, a carne equina apresentou maior teor de umidade. Assim, para a carne bovina já foram encontrados valores entre 72,48 e $74,48 \%$ (Almeida et al., 2006) e para carne de ovinos de 70,81 a 76,07\% (Madruga et al., 2005).
Variações na umidade estão relacionadas com a idade dos animais. Sarries \& Beriain (2005), afirmam que o maior teor de umidade e a maior atividade da água das carnes são encontrados em animais mais jovens do que em animais adultos. Assim, o percentual de água nos músculos diminui com o aumento da idade devido ao aumento da concentração de proteínas e de gorduras caracterizado pelo crescimento do animal. Por outro lado, o aumento do teor de lipídios não depende, necessariamente, do crescimento muscular, e sim da dieta do animal (Prandl et al., 1994).

O teor médio de lipídeos dos músculos analisados foi de $1.13 \%$, variando de 1,04 a $1,19 \%$. Esses valores estão em concordância com os resultados dos os músculos Longissumus dorsi $(1,02$ a $2,03 \%)$ e semimembranosus $(0,50$ a $1,55 \%$ ) obtidos por Junqueira et al. (2005), que

Tabela 1. Composição centesimal dos diferentes cortes cárneos de equinos machos.

\begin{tabular}{|c|c|c|c|c|c|}
\hline \multirow{2}{*}{ Corte Cárneo } & \multirow{2}{*}{ Animal } & \multicolumn{4}{|c|}{ Análise (G\%) } \\
\hline & & Umidade & Lipídios & Proteína & Cinzas \\
\hline \multirow{8}{*}{ 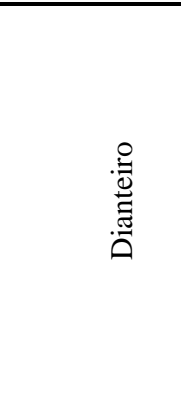 } & MD1 & $77,57 \pm 0,48^{\mathrm{A}, \mathrm{B}}$ & $1,09 \pm 0,13^{\mathrm{A}, \mathrm{B}}$ & $18,89 \pm 0,15^{\mathrm{B}}$ & $1,10 \pm 0,12^{\mathrm{A}}$ \\
\hline & MD2 & $79,15 \pm 0,25^{\mathrm{A}}$ & $1,23 \pm 0,03^{\mathrm{A}}$ & $23,04 \pm 0,89^{\mathrm{A}}$ & $1,18 \pm 0,02^{\mathrm{A}, \mathrm{B}}$ \\
\hline & MD3 & $77,91 \pm 0,53^{\mathrm{A}}$ & $0,85 \pm 0,05^{\mathrm{C}}$ & $19,31 \pm 0,30^{\mathrm{B}}$ & $1,06 \pm 0,02^{\mathrm{B}, \mathrm{C}}$ \\
\hline & MD4 & $76,03 \pm 0,33^{\mathrm{B}}$ & $0,92 \pm 0,01^{\mathrm{B}, \mathrm{C}}$ & $17,76 \pm 1,07^{\mathrm{B}}$ & $1,10 \pm 0,03^{\mathrm{B}, \mathrm{C}}$ \\
\hline & MD5 & $78,58 \pm 0,87^{\mathrm{A}}$ & $1,21 \pm 0,14^{\mathrm{A}}$ & $19,80 \pm 1,08^{\mathrm{B}}$ & $0,97 \pm 0,12^{\mathrm{C}}$ \\
\hline & MD6 & $78,61 \pm 1,38^{\mathrm{A}}$ & $1,01 \pm 0,18^{\mathrm{A}, \mathrm{B}, \mathrm{C}}$ & $18,73 \pm 1,96^{\mathrm{B}}$ & $1,14 \pm 0,05^{\mathrm{A}, \mathrm{B}}$ \\
\hline & MD7 & $77,45 \pm 1,02^{\mathrm{A}, \mathrm{B}}$ & $1,16 \pm 0,10^{\mathrm{A}}$ & $19,39 \pm 1,35^{\text {В }}$ & $1,29 \pm 0,07^{\mathrm{A}}$ \\
\hline & Média geral & $77,90 \pm 1,20^{\mathrm{A}}$ & $1,07 \pm 0,17^{\mathrm{A}}$ & $19,57 \pm 1,87^{\mathrm{A}}$ & $1,12 \pm 0,12^{\mathrm{A}}$ \\
\hline \multirow{8}{*}{$\begin{array}{l}\stackrel{0}{0} \\
\stackrel{0}{0}\end{array}$} & MDO1 & $78,84 \pm 0,35$ & $1,21 \pm 0,14^{\mathrm{A}, \mathrm{B}}$ & $16,75 \pm 0,95^{\mathrm{C}}$ & $1,21 \pm 0,16^{\mathrm{C}}$ \\
\hline & MDO2 & $81,24 \pm 0,90$ & $1,22 \pm 0,24^{\mathrm{A}, \mathrm{B}}$ & $19,85 \pm 2,28^{\mathrm{B}, \mathrm{C}}$ & $1,35 \pm 0,08^{\mathrm{B}, \mathrm{C}}$ \\
\hline & MDO3 & $77,06 \pm 1,28$ & $1,17 \pm 0,04^{\mathrm{a} \mathrm{B}}$ & $24,64 \pm 1,44^{\mathrm{A}}$ & $4,46 \pm 0,13^{\mathrm{A}}$ \\
\hline & MDO4 & $72,90 \pm 0,99$ & $1,51 \pm 0,40^{\mathrm{A}}$ & $24,07 \pm 3,68^{\mathrm{A}}$ & $0,94 \pm 0,09^{\mathrm{D}}$ \\
\hline & MDO5 & $80,15 \pm 1,19$ & $0,91 \pm 0,10^{\mathrm{B}}$ & $17,37 \pm 2,19^{\mathrm{C}}$ & $1,47 \pm 0,17^{\mathrm{B}}$ \\
\hline & MDO6 & $80,06 \pm 0,46$ & $0,99 \pm 0,05^{\mathrm{B}}$ & $23,62 \pm 0,66^{\mathrm{A}, \mathrm{B}}$ & $1,53 \pm 0,09^{\mathrm{B}}$ \\
\hline & MDO7 & $79,52 \pm 0,37$ & $1,00 \pm 0,01^{\mathrm{B}}$ & $18,19 \pm 1,22^{\mathrm{C}}$ & $1,43 \pm 0,09^{\mathrm{B}, \mathrm{C}}$ \\
\hline & Média geral & $78,54 \pm 2,75^{\mathrm{A}}$ & $1,14 \pm 0,26^{\mathrm{A}}$ & $20,64 \pm 3,68^{\mathrm{A}}$ & $1,77 \pm 1,14^{\mathrm{A}}$ \\
\hline \multirow{7}{*}{ 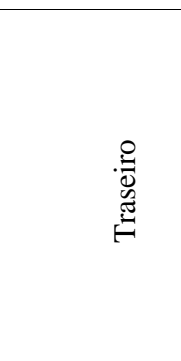 } & MT1 & $78,71 \pm 0,69^{\mathrm{A}, \mathrm{B}}$ & $1,18 \pm 0,16^{\mathrm{A}, \mathrm{B}}$ & $17,88 \pm 1,01^{\mathrm{B}}$ & $1,51 \pm 1,33^{\mathrm{A}, \mathrm{B}}$ \\
\hline & MT2 & $77,38 \pm 1,22^{\mathrm{B}}$ & $1,33 \pm 0,00^{\mathrm{A}}$ & $17,70 \pm 0,61^{\mathrm{B}}$ & $0,73 \pm 0,09^{\mathrm{B}}$ \\
\hline & MT3 & $79,54 \pm 0,49^{\mathrm{A}}$ & $1,13 \pm 0,05^{\mathrm{B}, \mathrm{C}}$ & $18,48 \pm 2,44^{\mathrm{B}}$ & $2,09 \pm 0,30^{\mathrm{A}}$ \\
\hline & MT4 & $79,19 \pm 0,12^{\mathrm{A}}$ & $1,29 \pm 0,01^{\mathrm{A}, \mathrm{B}}$ & $17,96 \pm 1,57^{\mathrm{B}}$ & $1,12 \pm 0,13^{\mathrm{A}, \mathrm{B}}$ \\
\hline & MT5 & $78,10 \pm 0,69^{\mathrm{A}, \mathrm{B}}$ & $1,24 \pm 0,00^{\mathrm{A}, \mathrm{B}}$ & $22,14 \pm 2,43^{\mathrm{A}}$ & $1,20 \pm 0,12^{\mathrm{A}, \mathrm{B}}$ \\
\hline & MT6 & $77,49 \pm 0,96^{\mathrm{B}}$ & $0,97 \pm 0,14^{\mathrm{C}}$ & $16,22 \pm 0,41^{\mathrm{B}}$ & $1,00 \pm 0,11^{\mathrm{A}, \mathrm{B}}$ \\
\hline & Média geral & $78,39 \pm 1,09^{\mathrm{A}}$ & $1,19 \pm 0,15^{\mathrm{A}}$ & $18,39 \pm 2,36^{\mathrm{A}}$ & $1,28 \pm 0,68^{\mathrm{A}}$ \\
\hline
\end{tabular}

*Médias seguidas pela mesma letra minúscula, na coluna, para o mesmo corte cárneo, são estatisticamente iguais pelo teste de Tukey a 5\% de significância. ** Médias seguidas pela mesma letra maiúscula, na coluna, para diferentes cortes cárneos, são estatisticamente iguais pelo teste de Tukey a 5\% de significância. 
verificaram ainda, que o peso ao abater e o sexo do animal não exerceram influência significativa sobre 0 teor de gordura destes músculos. Entretanto, os resultados obtidos nesse estudo são inferiores aos observados por Znamirowska \& Stanislawczyk (2005), que relatam valor médio de 2,57\%, para o músculo Longissumus dorsi, de cavalos jovens. Provavelmente, estas diferenças relacionam-se ao fato de que, no Brasil, são abatidos animais velhos e de descarte, com baixo escore corporal, enquanto que em alguns países europeus já existem criadores especializados na produção de equinos para abate.

A carne de cavalo apresenta um teor de lipídios menor do que outras espécies. Desta maneira, Almeida et al. (2006) encontraram valores de 3,08 e $8,75 \%$ nos músculos Bíceps femoris e semimembranosus de bovinos, respectivamente. Já para suínos, esses valores podem oscilar entre 3,2 a 5,0\% (Bragagnolo et al., 2002a, b). Em ovinos Madruga et al. (2005) encontraram valores entre 2,74 a $8,38 \%$. Na carne caprina, o teor pode variar de 2,51 a 3,18\% (Madruga et al., 2000).

Forrest et al. (1979) afirmam que o lipídeo é o componente químico mais variável dos músculos e do organismo animal, pois o aumento de lipídeos não depende necessariamente do crescimento muscular, e sim da dieta nutricional. Assim, o teor de ácidos graxos dos diferentes tipos de carnes é influenciado por uma grande variedade de fatores, incluindo raça do animal, teor de gordura intra e extramuscular, clima, condições de manejo e alimentação. Estes fatores também podem variar de acordo com a região onde os animais são criados e com as práticas culturais de cada região (Almeida et al., 2006).

Com relação ao teor de proteínas, foi obtido um valor médio de 19,53\%. Esses valores foram semelhantes nos três músculos analisados $(\mathrm{P}>0,05)$, como no estudo de Lorenzo et al. (2013a) que obtiveram teor de $22 \%$. Lorenzo et al. (2013b) realizaram avaliação da composição de aminoácidos na carne de potro, e afirmam que ela é uma ótima fonte de proteínas de alto valor biológico para a dieta, porque ela contém aminoácidos essenciais em uma proporção adequada, favorecendo a absorção de minerais, como ferro e os oligoelementos.

Contudo, comparando com outras espécies, o teor de proteínas da paleta $(19,57 \%)$ e do contrafilé $(20,64 \%)$ é similar à média encontrada para ovinos (19,59 a 21,06\%) por Madruga et al.
(2005). A porcentagem de proteína encontrada para o contra filé do presente estudo também é semelhante à do contrafilé bovino (20,58 a $21,14 \%)$ obtida por Moreira et al. (2003).

Assim como teor de umidade, lipídeos e proteínas, a porcentagem de cinzas foi semelhante para todas as amostras, sendo a média de 1,39\%. Lorenzo et al. (2013a) obtiveram como média $(1.26 \%)$ para o teor de cinzas nos diferentes músculos e também não observaram diferença significativa entre as amostras $(\mathrm{P}>0,05)$. Outros estudos obtiveram resultados inferiores e superiores ao obtido neste estudo para o teor de cinzas na carne de cavalo, como é o caso de Franco \& Lorenzo (2014) (1.22\%); e Sarriés \& Beriain (2005), 2,56 e 4,03\% Essa variabilidade de resultados dos teores de cinzas é devido à variação do conteúdo mineral das pastagens que cada animal consome (Lorenzo et al., 2014). Variações na composição química de carnes também podem ocorrer devido à influência hormonal, estando diretamente relacionada com prática de castração de animais para abate (Padre et al., 2006).

\section{Conclusão}

Não foram observadas diferenças entre a composição química dos diferentes cortes cárneos de equinos machos. A carne de cavalo caracterizou-se por altos teores de umidade e de proteína e baixos de lipídeos.

\section{Referências bibliográficas}

Almeida, J. C., Perassolo, M. S., Camargo, J. L., Bragagnolo, N. \& Gross, J. L. 2006. Composição de ácidos graxos e conteúdo de colesterol de cortes de carne de gado e frango do Sul do Brasil. Revista Brasileira de Ciências Farmacêuticas, 42,109-117.

Bragagnolo, N. \& Rodriguez-Amaya, D. B. 2002a. Simultaneous determination of total lipid, cholesterol and fatty acids in meat and backfat of suckling and adult pigs. Food Chemistry, 79, 255-260.

Bragagnolo, N. \& Rodriguez-Amaya, D. B. 2002b. Teores de colesterol, lipídios totais e ácidos graxos em cortes de carne suína. Ciência e Tecnologia de Alimentos, 22, 98104.

Brasil, Ministério da Agricultura, Pecuária e Abastecimento. 1999. Instrução Normativa n. 20, de 21 de julho de 1999. Métodos analíticos oficiais físico-químicos para controle de carnes, produtos cárneos e seus ingredientes, 
sal e salmoura. Diário Oficial da República Federativa do Brasil, Brasília, DF.

Costa, M. D.; Mendes, L. J.; Maruch, S., Ramirez, P. A., Meneses, A. C. A., Neto, T. M., Ruas, J. R. M. \& Chamone, J. M. A. 2016. Efeito da composição genética nas características de conformação em equinos. Arquivo Brasileiro de Medicina Veterinária e Zootecnia, 68, 1629-1637.

Forrest, J. C. 1979. Fundamentos de ciência de la carne. Zaragoza: ACRIBIA.

Franco, D. \& Lorenzo, J. M. 2014. Effect of muscle and intensity of finishing diet on meat quality of foals slaughtered at 15 months. Meat Science, 96, 327-334.

Juárez, M.; Polvillo, O.; Gómez, M. D., Alcalde, M. J., Romero, F. \& Valera, M. 2009. Breed effect on carcass and meat quality of foals slaughtered at 24 months of age. Meat Science, $83,224-228$.

Junqueira, A. C. A., Bressan, M. C., Rebello, F. F. P., Faria, P. B., Vieira, J. O. \& Savian, T. V. 2005. Composição centesimal e teor de colesterol na carne de eqüinos (Equus caballus, Linneaus, 1758) machos e fêmeas, agrupados por peso de carcaça. Ciência e Agrotecnologia, $29,362-368$.

Lorenzo, J. M., Pateiro, M. \& Franco, D. 2013a. Influence of muscle type on physicochemical and sensory properties of foal meat. Meat Science, 94, 77-83.

Lorenzo, J. M., Sarriés, M. V. \& Franco, D. 2013b. Sex effect on meat quality and carcass traits of foals slaughtered at 15 months of age. Animal, 7, 199-1207.

Lorenzo, J. M., Sarriés, M. V., Tateo, A., Polidori, P., Franco, D. \& Lanza, M. 2014. Carcass characteristics, meat quality and nutritional value of horsemeat: A review. Meat Science, 96, 1478-1488.

Madruga, M. S., Arruda, S. G. B., Andrade, L. T. \& Beserra, F. J. 2000. Efeito da castração sobre parâmetros químicos, físico-químicos e sensoriais da carne caprina de animais mestiços. Ciência e Tecnologia de Alimentos, 20, 23-26.

Madruga, M. S., Sousa, W. H., Rosales, M. D., Cunha, M. G. G. \& Ramos, J. L. F. 2005. Qualidade da carne de cordeiros Santa Inês terminados com diferentes dietas. Revista Brasileira de Zootecnia, 34, 309-315.

Moreira, F. B., Souza, N. E. De Matsushita, M., Prado, I. N. \& Nascimento, W. G. 2003. Evaluation of carcass characteristics and meat chemical composition of Bos indicus and Bos indicus $\mathrm{x}$ Bos taurus crossbred steers finished in pasture systems. Brazilian Archives of Biology and Technology, 46, 609-616.

Padre, R. G., Aricetti, J. A., Moreira, F. B., Mizubuti, I. Y., Do Prado, I. N.,Visentainer, J. V., De Souza, N. E. \& Matsushita, M. 2006. Fatty acid profile and chemical composition of Longissimus muscle of bovine steers and bulls finished in pasture system. Meat Science, 74, 242-248.

Prandl, O., Fischer, A., Schmidhofer, T. \& Sinell, H. J. 1994. Tecnología e higiene de la carne. Zaragoza: ACRIBIA.

R Development Core Team. R: A language and environment for statistical computing. $\mathrm{R}$ Foundation Statistical Computing, 2006. Disponível em: <http://www.R-project.org>. Acessado em: 15 agost. 2017.

Sarriés, M. V. \& Beriain, M. J. 2005. Carcass characteristics and meat quality ofmale and female foals. Meat Science, 70, 141-152.

Stanciu, S. 2015. Horse Meat ConsumptionBetween Scandal and Reality. Procedia Economics and Finance. 23, 697-703.

Tateo, A., De Palo, P. \& Centoducati P. 2008. Physicochemical properties of meat of Italian Heavy Draft horses slaughtered at the age of 11 months. Journal of Animal Science,86, 12051214

Znamirowska, A. \& Stanislawczyk, R. 2005. Effect of maturing process on changes in physico-chemical properties of colt meat. Acta Scientiarum Polonorum-Technologia Alimentaria, 4, 79-88.

Article History:

Received 15 March 2018

Accepted 13 April 2018

Available online 31 May 2018

License information: This is an open-access article distributed under the terms of the Creative Commons Attribution License 4.0, which permits unrestricted use, distribution, and reproduction in any medium, provided the original work is properly cited. 